\title{
Asymptotic behavior of the magnetization for the perceptron model
}

\author{
David Márquez-Carreras ${ }^{\mathrm{a}, 1}$, Carles Rovira ${ }^{\mathrm{a}, 2}$, Samy Tindel $^{\mathrm{b}, 3, *}$ \\ ${ }^{a}$ Facultat de Matemàtiques, Universitat de Barcelona, Gran Via 585, 08007-Barcelona, Spain \\ b Institut Élie Cartan, Université de Nancy 1, BP 239, 54506 Vandoeuvre-lès-Nancy, France
}

Received 8 December 2003; received in revised form 10 December 2004; accepted 15 April 2005

Available online 18 November 2005

\begin{abstract}
In this paper, we show that, in case of a perceptron model for which the number of outputs is a small proportion of the size of the system, the limiting behavior of the magnetization of a given spin, namely the random variable $\left\langle\sigma_{k}\right\rangle$, can be identified. In fact, we prove a $L^{2}$ convergence for a collection of those random variables.
\end{abstract}

๑) 2005 Elsevier SAS. All rights reserved.

\section{Résumé}

Dans cet article, nous montrons que, dans le cas d'un modèle du perceptron pour lequel le nombre de sorties est une proportion suffisamment petite de la taille du système, on peut identifier le comportement limite de la magnétisation d'un spin quelconque, c'est-à-dire de la variable aléatoire $\left\langle\sigma_{k}\right\rangle$. Nous montrons en fait une convergence dans $L^{2}$ pour une famille de telles variables.

(c) 2005 Elsevier SAS. All rights reserved.

MSC: 60G15; 82D30

Keywords: Spin glasses; Perceptron model; Cavity method

\section{Introduction}

One of the basic problems in neural computation theory is the supervised learning for pattern recognition by a simple perceptron (we refer to [2] and references therein for a detailed account on that theory, from a physical and numerical point of view). This problem can be summarized in the following way: for two given integers $N, M \geqslant 1$ and $i \in\{1, \ldots, N\}$, the input of the perceptron is $\eta=\left(\eta_{1}, \ldots, \eta_{N}\right)$ with $\eta_{i} \in\{-1,1\}$, and its output is of the form

$$
O_{k}=u\left(\sum_{i \leqslant N} w_{i, k} \eta_{i}\right), \quad k \leqslant M,
$$

\footnotetext{
* Corresponding author.

E-mail addresses: marquez@mat.ub.es (D. Márquez-Carreras), carles.rovira@ub.edu (C. Rovira), tindel@iecn.u-nancy.fr (S. Tindel).

1 Partially supported by DGES grant BFM2000-0607.

2 Partially done while the author was visiting the Université Paris 13 with a CIRIT grant. Partially supported by DGES grant BFM2000-0607.

3 Partially written with a support of a fellowship grant at the Centre de Recerca Matemàtica (Bellaterra, Barcelona). The author would like to thank this institution for its warm hospitality.
} 
for a collection of real weights $\left\{w_{i, k} ; i \leqslant N, k \leqslant M\right\}$, and where $u: \mathbb{R} \rightarrow \mathbb{R}$ should be, for numerical purposes, a step function. Note however that, in many case, $u$ is taken as a regularization of the sign function, whose derivatives are allowed to grow at exponential speed with $N$. This is assumed for mathematical convenience, and also because it reproduces better the actual behavior of the brain synapses, from which this kind of model is inspired.

The supervised learning procedure consists then in providing $p$ inputs $\eta^{1}, \ldots, \eta^{p}$, jointly with $p$ desired output patterns $\zeta^{1}, \ldots, \zeta^{p}$ (called the training set), and, if possible, try to find the weights $w_{i, k}$ matching the inputs and the outputs, i.e. such that

$$
u\left(\sum_{i \leqslant N} w_{i, k} \eta_{i}^{l}\right)-\zeta_{k}^{l}=0, \quad k \leqslant M, l \leqslant p .
$$

Before computing those weights, a natural and classical question is to get some information about the theoretical capacity of our model, that is the maximal number of patterns for which the problem (1) can be solved. Without any a priori knowledge about $w_{i, k}$ and $\eta^{l}$, the following assumptions can be done:

1. The inputs $\eta$ are uniformly distributed in $\Sigma_{N}=\{-1,1\}^{N}$.

2. The hyperplanes defined by the weights $w_{i, k}$ are also taken as "uniformly" distributed random planes, which is equivalent to model $w_{i, k}$ as an i.i.d. family of standard Gaussian random variables, normalized by $N^{-1 / 2}$ in order to get a term of order 1 . That is, one chooses

$$
w_{i, k}=\frac{g_{i, k}}{N^{1 / 2}},
$$

where $\left\{g_{i, k} ; i \geqslant 1, k \geqslant 1\right\}$ is a family of independent standard Gaussian random variables.

However, it is a hard task to solve the system (1). Thus, in that context, one step further in the analysis is to replace it by the study of an associated Gibbs measure, a usual trick known as the finite temperature approximation. In our case, if one wishes to know on how many bits $M$ the output can be coded, a natural measure to consider is the one whose density with respect to the uniform measure $\mu_{N}$ on $\Sigma_{N}$ is given, for a typical configuration $\sigma=\left(\sigma_{1}, \ldots, \sigma_{N}\right) \in \Sigma_{N}$, by $Z_{N, M}^{-1} \exp \left(-H_{N, M}(\sigma)\right)$, with

$$
-H_{N, M}(\sigma)=\sum_{k \leqslant M} u\left(N^{-1 / 2} \sum_{i \leqslant N} g_{i, k} \sigma_{i}\right), \quad Z_{N, M}=\sum_{\sigma \in \Sigma_{N}} \exp \left(-H_{N, M}(\sigma)\right) .
$$

Notice that, in order to get the formula (2), one has to remove all the constant weights $\zeta_{k}^{l}$, and to include the usual inverse of the temperature parameter $\beta$ into the function $u$, which are also two standard tricks.

It is now easily seen that a phase diagram for the model given by (2), known as the perceptron model, would give some essential information about the capacity of the original model. This phase diagram is far from being complete from a mathematical point of view. However, the deep study initiated in [4, Chapter 3] (see also [3] in a slightly different context) leads to a rigorous replica symmetric solution when $M$ is a (small enough) proportion of $N$, i.e. $M=$ $\alpha N$ with $\alpha$ small enough. This replica symmetric solution indicates that the system does not behave too chaotically when $N \rightarrow \infty$, and hence that the capacity of the perceptron is not reached yet.

Another way of looking at the regularity of the system when $N \rightarrow \infty$ is to investigate the asymptotic behavior of the magnetization of an arbitrary family of spins $\sigma_{1}, \ldots, \sigma_{k}$ for $k \geqslant 1$, that is the limit of the laws of the random variables

$$
\left\langle\sigma_{1}\right\rangle, \ldots,\left\langle\sigma_{k}\right\rangle,
$$

where $\langle\cdot\rangle$ designates the average with respect to the measure defined by (2). In fact, in most engineering applications, the classical way to illustrate the self averaging phenomenon for this kind of model is to show that those quantities converge to some independent and identically distributed centered random variables that can be clearly identified, by analogy with the fact that the magnetization vanishes for the Ising model at high temperature. Therefore, our aim in this paper will be to give an answer to that natural problem, which can be summarized in the following theorem: 
Theorem 1.1. Assume u satisfies the assumptions

(H1) There exist a constant $D>0$ and a large enough number $L$ such that $|u(x)| \leqslant D$ for all $x$ and $L \mathrm{e}^{L D} \leqslant 1$.

(H2) There exists a positive constant $L^{*}$ and a small enough constant $c_{3}$ such that, for any $l \leqslant 12,\left|u^{(l)}\right| \leqslant$ $L^{*} \mathrm{e}^{L^{*} D} \mathrm{e}^{c_{3} N}$.

Then, there exists a constant $r \geqslant 0$, that will be defined at Section 2, such that, for any fixed $m \geqslant 1$,

$$
\sum_{i \leqslant m} \mathbf{E}\left[\left(\left\langle\sigma_{i}\right\rangle-\tanh \left(r^{1 / 2} z_{i}\right)\right)^{2}\right] \leqslant \frac{\kappa_{m}}{N^{1 / 2}}
$$

where $\kappa_{m}$ is a positive constant, and $\left(z_{1}, \ldots, z_{m}\right)$ is a family of independent standard Gaussian random variables (which still depend, however, of the randomness contained in $g$ ).

Let us give now some hints about the way one can get that result: of course, our proof will be inspired by the methods introduced in [4], and particularly by the smart path method, which is an efficient way of interpolating two Gaussian vectors, once the limiting local fields corresponding to (2) can be guessed. In our case, if the natural path is quite easy to figure out (see (8) for a heuristic justification), the computations along that path are long and sometimes involved. In particular, the path (18), which seems to be the obvious one at first sight, lead us to a series of technical problems. Like in [1], we have chosen to give some details of our calculations for sake of readability, hoping that the final aspect of our paper will not be too scary.

Notice also that the optimal expected rate of convergence in our theorem would be in $N^{-1}$. We stopped at $N^{-1 / 2}$ and did not check the computations allowing to get this optimal rate, but we believe that it could be reached along the same lines we have developed in this paper. In any case, let us recall again that our result answers a natural question for the perceptron model, and corresponds to one of the basic facts that practitioners are looking for, as mentioned in [2]. Observe also that our way to derive Theorem 1.1 is certainly not the only one, and we hope that, in the future, some easier proofs can be elaborated. And it is worth pointing out that the methods contained in [3] could potentially yield one of these simplifications. However, the latter paper is based on some convexity assumptions on the function $u$, and some spherical hypothesis on the spin model, which makes it hard to adapt to our situation.

Our paper is divided as follow: in the next section, we will introduce the basic notations concerning the perceptron model, and recall some results taken from [4]. Section 3 is devoted to state an important intermediate result (Proposition 3.1) on our way to the proof of Theorem 1.1, to the definition of the Gaussian path we will use in the sequel, and to the first computations along this path. Some new Gibbs measures appear naturally in those computations. We will introduce them at Section 4. The main step towards Theorem 1.1 will then be achieved with an asymptotic expansion of a general term involving the measures introduced at Section 4. This will be done at Section 5. Then, we will apply those results in order to prove Proposition 3.1 at Section 6. Theorem 1.1 will be proved at Section 7. Eventually, let us mention that, in the remainder of the paper, $\kappa, c, c_{1}, \ldots$ will stand for some positive constants, that can change from line to line.

\section{Preliminary results}

As mentioned in the introduction, the state space of our model will be $\Sigma_{N}=\{-1,1\}^{N}$ for a given $N \in \mathbb{N}^{*}$, and setting $\mu_{N}$ for the uniform measure on $\Sigma_{N}$, we will consider the random measure whose density with respect to $\mu_{N}$ is given by $Z_{N, M}^{-1} \exp \left(-H_{N, M}(\sigma)\right)$, where $Z_{N, M}$ has been introduced at relation (2), and where we recall that $H_{N, M}(\sigma)$ is defined by

$$
-H_{N, M}(\sigma)=\sum_{k \leqslant M} u\left(N^{-1 / 2} \sum_{i \leqslant N} g_{i, k} \sigma_{i}\right) .
$$

In the above formula, $M$ stands for a positive integer such that $M=\alpha N$ for a given $\alpha>0, u$ will be a continuous function defined on $\mathbb{R}$ satisfying conditions (H1) and (H2) given in the introduction, and $\left\{g_{i, k} ; i \geqslant 1, k \geqslant 1\right\}$ is a family of independent standard Gaussian random variables. Note that $u$ and $\alpha$ will depend on $N$, though this dependence will be kept implicit for sake of readability. 
We will first give an account on the results obtained in [4] concerning the perceptron model. Those results are based on the smart path method, that is a way to single out continuously the last spin $\sigma_{N}$ from the other ones in the definition of $H_{N, M}$. This smart path can be described as follows: for $t \in[0,1], \sigma \in \Sigma_{N}$, set

$$
S_{k, t}(\sigma)=\left(\frac{1}{N}\right)^{1 / 2} \sum_{i \leqslant N-1} g_{i, k} \sigma_{i}+\left(\frac{t}{N}\right)^{1 / 2} g_{N, k} \sigma_{N},
$$

and consider the Hamiltonian

$$
-H_{N, M, t}(\sigma)=\sum_{k \leqslant M} u\left(S_{k, t}(\sigma)\right)+\sigma_{N}(r(1-t))^{1 / 2} Y,
$$

where $r$ is a positive coefficient whose exact value will be given later on, and $Y$ is a standard Gaussian random variable independent of all the other randomness in $H_{N, M}$. For a given function $f: \Sigma_{N}^{n} \rightarrow \mathbb{R}$, set now $\langle f\rangle_{t}$ for the Gibbs mean of $f$ with respect to the Hamiltonian $H_{N, M, t}$, and

$$
v_{t}(f)=\mathbf{E}\left[\langle f\rangle_{t}\right] \text {. }
$$

Then, on one hand, some simplifications in the computation of $v_{t}(f)$ can be done when $t=0$ :

Lemma 2.1. Let $f^{-}$be a function defined on $\Sigma_{N-1}^{n}$, and I be a subset of $\{1, \ldots, n\}$ of cardinality $|I|$. Then

$$
v_{0}\left(f^{-} \prod_{l \in I} \sigma_{N}^{l}\right)=\mathbf{E}\left[\left(\tanh \left(r^{1 / 2} Y\right)\right)^{|I|}\right] \nu_{0}\left(f^{-}\right)
$$

Notice that, in the previous result, $\sigma^{1}, \ldots, \sigma^{n}$ are understood as $n$ independent configurations under $G_{N}$, usually called replicas of $\sigma$.

On the other hand, the derivative of $v_{t}(f)$ with respect to the parameter $t$ can be computed explicitly.

Proposition 2.2. Suppose that $u$ is twice differentiable. Write $w=u^{\prime \prime}+\left(u^{\prime}\right)^{2}$. Then, for $0<t<1$ and $f: \Sigma_{N}^{n} \rightarrow \mathbb{R}$, we have

$$
v_{t}^{\prime}(f)=\sum_{j=1}^{3} K_{j}
$$

where

$$
K_{1}=\frac{\alpha}{2} \sum_{l \leqslant n} v_{t}\left(w\left(S_{M, t}^{l}\right) f\right)-\frac{\alpha}{2} n v_{t}\left(w\left(S_{M, t}^{n+1}\right) f\right),
$$

$K_{2}$ is defined by

$$
\begin{aligned}
K_{2}= & \alpha \sum_{1 \leqslant l<l^{\prime} \leqslant n} v_{t}\left(\sigma_{N}^{l} \sigma_{N}^{l^{\prime}} u^{\prime}\left(S_{M, t}^{l}\right) u^{\prime}\left(S_{M, t}^{l^{\prime}}\right) f\right)-\alpha n \sum_{l \leqslant n} v_{t}\left(\sigma_{N}^{l} \sigma_{N}^{n+1} u^{\prime}\left(S_{M, t}^{l}\right) u^{\prime}\left(S_{M, t}^{n+1}\right) f\right) \\
& +\alpha \frac{n(n+1)}{2} v_{t}\left(\sigma_{N}^{n+1} \sigma_{N}^{n+2} u^{\prime}\left(S_{M, t}^{n+1}\right) u^{\prime}\left(S_{M, t}^{n+2}\right) f\right),
\end{aligned}
$$

and

$$
K_{3}=-r\left(\sum_{1 \leqslant l<l^{\prime} \leqslant n} v_{t}\left(\sigma_{N}^{l} \sigma_{N}^{l^{\prime}} f\right)-n v_{t}\left(\sigma_{N}^{l} \sigma_{N}^{n+1} f\right)+\frac{n(n+1)}{2} v_{t}\left(\sigma_{N}^{n+1} \sigma_{N}^{n+2} f\right)\right) .
$$

The drawback of Proposition 2.2 is that some terms involving $u^{\prime}$ and $u^{\prime \prime}$ appear in the formula, while one would like to have only a mild control on those quantities. However, using some integration by parts arguments, some asymptotic results for the perceptron model are obtained in [4]: first of all, define, for two replicas $\sigma^{l}, \sigma^{l^{\prime}}$ of $\sigma$, the quantity

$$
R_{l, l^{\prime}}=\frac{1}{N} \sum_{i \leqslant N} \sigma_{i}^{l} \sigma_{i}^{l^{\prime}}
$$


This quantity, called the overlap of $\sigma^{l}$ and $\sigma^{l^{\prime}}$, will be, as usual in the spin glass theory, a central object in the study of the model. It is then essential to obtain some sharp results on the asymptotic behavior of $R_{1,2}$, that we will describe now: let $q$ and $r$ (notice that $r$ has already been introduced in (4)) be the solution to the system

$$
q=\mathbf{E}\left[\tanh ^{2}\left(r^{1 / 2} Z\right)\right], \quad r=\alpha \mathbf{E}\left[\Lambda^{2}\left(q^{1 / 2} Z,(1-q)^{1 / 2}\right)\right],
$$

where $\Lambda$ is defined on $\mathbb{R}^{2}$ by

$$
\Lambda(x, y):=\frac{\mathbf{E}[\xi \exp (u(x+\xi y))]}{y \mathbf{E}[\exp (u(x+\xi y))]}=\frac{\mathbf{E}\left[u^{\prime}(x+\xi y) \exp (u(x+\xi y))\right]}{\mathbf{E}[\exp (u(x+\xi y))]},
$$

and where the second equality is obtained by a Gaussian integration by parts. In the preceding formulae, $Z$ and $\xi$ stand for two independent standard Gaussian random variables. Observe that, under hypothesis (H1), the system (5) admits a unique solution. Then the following inequalities hold true (notice that these inequalities are shown in [4] under slightly weaker assumptions):

Proposition 2.3. Under hypothesis $(\mathrm{H} 1)$ and $(\mathrm{H} 2)$, we have, for any $k \geqslant 0$,

$$
v\left(\left(R_{1,2}-q\right)^{2 k}\right) \leqslant\left(\frac{L k}{N}\right)^{k} .
$$

Furthermore, we have

$$
\left|v\left(R_{1,2}-q\right)\right| \leqslant \frac{L}{N}
$$

Notice that the computations leading to this exponential self-averaging property for the overlap also yield the replica symmetric solution for the model. We refer to [4] for this last formula.

Let us end this section by recalling an elementary integration by parts formula we will use throughout the paper: for $p \geqslant 1$, let $\left(g_{1}, \ldots, g_{p}\right)$ be a Gaussian vector in $\mathbb{R}^{p}$, and $F: \mathbb{R}^{p} \rightarrow \mathbb{R}$ be a $C^{1}$ function having at most exponential growth together with its gradient. Then

$$
\mathbf{E}\left[g_{1} F\left(g_{1}, \ldots, g_{m}\right)\right]=\sum_{l \leqslant m} \mathbf{E}\left[g_{1} g_{l}\right] \mathbf{E}\left[\partial_{x_{l}} F\left(g_{1}, \ldots, g_{m}\right)\right] .
$$

\section{Definition of the path}

A first step in order to prove Theorem 1.1 is to achieve an asymptotic decomposition of $\left\langle\sigma_{N}\right\rangle$ separating, on one hand, the randomness contained in $\left\{g_{N, k} ; k \leqslant M\right\}$, and on the other hand, some terms involving $\left\{g_{i, k} ; i \leqslant N-1\right.$, $k \leqslant M\}$.

First of all let us introduce a new Hamiltonian

$$
-H_{N-1, M}(\sigma)=\sum_{k \leqslant M} u\left(N^{-1 / 2} \sum_{i \leqslant N-1} g_{i, k} \sigma_{i}\right)=\sum_{k \leqslant M} u\left(S_{k, 0}\right),
$$

where the quantities $S_{k, t}$ have been defined at (3), and let $\langle\cdot\rangle_{-}$denote the average with respect to the Gibbs measure induced by $H_{N-1, M}$.

Then, the following heuristic steps can be performed: we have

$$
-H_{N, M}(\sigma) \simeq-H_{N-1, M}(\sigma)+N^{-1 / 2} \sum_{k \leqslant M} u^{\prime}\left(S_{k, 0}\right) g_{N, k} \sigma_{N},
$$

and hence

$$
\left\langle\sigma_{N}\right\rangle \simeq \frac{\left\langle\sinh \left(\left(1 / N^{1 / 2}\right) \sum_{k \leqslant M} g_{N, k} u^{\prime}\left(S_{k, 0}\right)\right)\right\rangle_{-}}{\left\langle\cosh \left(\left(1 / N^{1 / 2}\right) \sum_{k \leqslant M} g_{N, k} u^{\prime}\left(S_{k, 0}\right)\right)\right\rangle_{-}} .
$$

We will show in the sequel that this crude estimate holds true in the limit $N \rightarrow \infty$, and in fact, using a direct analogy with the self averaging properties of the SK model, we will prove the following result: 
Proposition 3.1. Under hypothesis (H1) and (H2), there exists a strictly positive constant $\kappa$ such that

$$
\mathbf{E}\left[\left(\left\langle\sigma_{N}\right\rangle-\tanh \left(\frac{1}{N^{1 / 2}} \sum_{k \leqslant M} g_{N, k}\left\langle u^{\prime}\left(S_{k, 0}\right)\right\rangle_{-}\right)\right)^{2}\right] \leqslant \frac{\kappa}{N^{1 / 2}} .
$$

Most of the remainder of the paper will be devoted to prove (9), and let us begin here with some simple considerations, that is, the definition of the Gaussian path we will use: for $t \in[0,1]$, let us consider the function

$$
\varphi(t)=\mathbf{E}\left[\left(\left\langle\sigma_{N}\right\rangle_{t}-\tanh Y_{t}\right)^{2}\right],
$$

where

$$
Y_{t}=\left(\frac{t}{N}\right)^{1 / 2} \sum_{k \leqslant M} g_{N, k}\left\langle u^{\prime}\left(S_{k, 0}\right)\right\rangle_{-}+[r(1-t)]^{1 / 2} Y .
$$

Notice then that $\varphi(1)$ is the left-hand side of (9), and on the other hand, using that $\left\langle\sigma_{N}\right\rangle_{0}=\tanh \left(r^{1 / 2} Y\right)$, it is easy to check that $\varphi(0)=0$. By means of the elementary equation

$$
\varphi(1)-\varphi(0)=\int_{0}^{1} \varphi^{\prime}(t) \mathrm{d} t
$$

we can see that (9) will be achieved as soon as we can show that

$$
\left|\varphi^{\prime}(t)\right| \leqslant \frac{\kappa}{N^{1 / 2}},
$$

for any $t \in(0,1)$. Hence, our first task will be to compute the derivative of $\varphi$. In fact, we will start by decomposing $\varphi$ into three terms:

$$
\varphi(t)=\varphi_{1}(t)+\varphi_{2}(t)+\varphi_{3}(t),
$$

with

$$
\begin{aligned}
\varphi_{1}(t) & =\mathbf{E}\left[\left\langle\sigma_{N}\right\rangle_{t}^{2}\right], \\
\varphi_{2}(t) & =\mathbf{E}\left[\tanh ^{2}\left(Y_{t}\right)\right], \\
\varphi_{3}(t) & =-2 \mathbf{E}\left[\left\langle\sigma_{N}\right\rangle_{t} \tanh Y_{t}\right] .
\end{aligned}
$$

Then, we will compute the derivatives of $\varphi_{1}, \varphi_{2}$ and $\varphi_{3}$ separately.

Using two replicas of $\sigma$, we get $\varphi_{1}(t)=v_{t}\left(\sigma_{N}^{1} \sigma_{N}^{2}\right)$. Then, as a direct consequence of Proposition 2.2, we get the following lemma.

Lemma 3.2. For any $t \in[0,1]$, we have

$$
\varphi_{1}^{\prime}(t)=\sum_{k \leqslant 6} A_{1, k}(t)
$$

with

$$
\begin{aligned}
& A_{1,1}(t)=\alpha v_{t}\left[\left(u^{\prime \prime}+\left(u^{\prime}\right)^{2}\right)\left(S_{M, t}^{1}\right) \sigma_{N}^{1} \sigma_{N}^{2}\right], \\
& A_{1,2}(t)=-\alpha v_{t}\left[\left(u^{\prime \prime}+\left(u^{\prime}\right)^{2}\right)\left(S_{M, t}^{3}\right) \sigma_{N}^{1} \sigma_{N}^{2}\right], \\
& A_{1,3}(t)=\alpha v_{t}\left[u^{\prime}\left(S_{M, t}^{1}\right) u^{\prime}\left(S_{M, t}^{2}\right)\right], \\
& A_{1,4}(t)=-4 \alpha v_{t}\left[u^{\prime}\left(S_{M, t}^{1}\right) u^{\prime}\left(S_{M, t}^{3}\right) \sigma_{N}^{2} \sigma_{N}^{3}\right], \\
& A_{1,5}(t)=3 \alpha v_{t}\left[u^{\prime}\left(S_{M, t}^{3}\right) u^{\prime}\left(S_{M, t}^{4}\right) \sigma_{N}^{1} \sigma_{N}^{2} \sigma_{N}^{3} \sigma_{N}^{4}\right], \\
& A_{1,6}(t)=-r\left[1-4 v_{t}\left(\sigma_{N}^{1} \sigma_{N}^{2}\right)+3 v_{t}\left(\sigma_{N}^{1} \sigma_{N}^{2} \sigma_{N}^{3} \sigma_{N}^{4}\right)\right] .
\end{aligned}
$$

Differentiating the function $\varphi_{2}(t)$ and using the integration by parts formula (7), we also obtain the following result. 
Lemma 3.3. For any $t \in[0,1]$, we have

$$
\varphi_{2}^{\prime}(t)=A_{2,1}(t)+A_{2,2}(t),
$$

with

$$
\begin{aligned}
& A_{2,1}(t)=\alpha \mathbf{E}\left[\left(1-\tanh ^{2}\left(Y_{t}\right)\right)\left(1-3 \tanh ^{2}\left(Y_{t}\right)\right)\left\langle\left. u^{\prime}\left(S_{M, 0}\right)\right|_{-} ^{2}\right],\right. \\
& A_{2,2}(t)=-r \mathbf{E}\left[\left(1-\tanh ^{2}\left(Y_{t}\right)\right)\left(1-3 \tanh ^{2}\left(Y_{t}\right)\right)\right] .
\end{aligned}
$$

Now, differentiating $\varphi_{3}(t)$ and using again the integrate by parts formula (7) we get the next lemma.

Lemma 3.4. For any $t \in[0,1]$, we have

$$
\varphi_{3}^{\prime}(t)=-2\left(\sum_{i \leqslant 4} A_{3,1,1, i}(t)-\sum_{i \leqslant 5} A_{3,1,2, i}(t)+A_{3,2,1}(t)-A_{3,2,2}(t)\right),
$$

with

$$
\begin{aligned}
& A_{3,1,1,1}(t)=\frac{\alpha}{2} \mathbf{E}\left[\left\langle\sigma_{N}\left(u^{(2)}\left(S_{M, t}\right)+\left(u^{\prime}\left(S_{M, t}\right)\right)^{2}\right)\right\rangle_{t} \tanh Y_{t}\right], \\
& A_{3,1,1,2}(t)=-\frac{\alpha}{2} \mathbf{E}\left[\left\langle\sigma_{N}^{2} u^{\prime}\left(S_{M, t}^{1}\right) u^{\prime}\left(S_{M, t}^{2}\right)\right\rangle_{t} \tanh Y_{t}\right], \\
& A_{3,1,1,3}(t)=\frac{\alpha}{2} \mathbf{E}\left[\left\langle u^{\prime}\left(S_{M, t}\right)\right\rangle_{t}\left\langle u^{\prime}\left(S_{M, 0}\right)\right\rangle_{-}\left(1-\tanh ^{2}\left(Y_{t}\right)\right)\right], \\
& A_{3,1,1,4}(t)=-\frac{r}{2} \mathbf{E}\left[1-\tanh ^{2}\left(Y_{t}\right)\right],
\end{aligned}
$$

the terms $A_{3,1,2, i}(t)$ being defined by

$$
\begin{aligned}
& A_{3,1,2,1}(t)=\frac{\alpha}{2} \mathbf{E}\left[\left\langle\sigma_{N}^{1}\left(u^{(2)}\left(S_{M, t}^{2}\right)+\left(u^{\prime}\left(S_{M, t}^{2}\right)\right)^{2}\right)\right\rangle_{t} \tanh Y_{t}\right], \\
& A_{3,1,2,2}(t)=\frac{\alpha}{2} \mathbf{E}\left[\left\langle\sigma_{N}^{1} u^{\prime}\left(S_{M, t}^{1}\right) u^{\prime}\left(S_{M, t}^{2}\right)\right\rangle_{t} \tanh Y_{t}\right], \\
& A_{3,1,2,3}(t)=-\alpha \mathbf{E}\left[\left\langle\sigma_{N}^{1} \sigma_{N}^{2} \sigma_{N}^{3} u^{\prime}\left(S_{M, t}^{1}\right) u^{\prime}\left(S_{M, t}^{2}\right)\right\rangle_{t} \tanh Y_{t}\right], \\
& A_{3,1,2,4}(t)=\frac{\alpha}{2} \mathbf{E}\left[\left\langle\sigma_{N}^{1} \sigma_{N}^{2} u^{\prime}\left(S_{M, t}^{2}\right)\right\rangle_{t}\left\langle u^{\prime}\left(S_{M, 0}\right)\right\rangle_{-}\left(1-\tanh ^{2} Y_{t}\right)\right], \\
& A_{3,1,2,5}(t)=-r \mathbf{E}\left[\left\langle\sigma_{N}\right\rangle_{t} \tanh Y_{t}\right]+r \mathbf{E}\left[\left\langle\sigma_{N}^{1} \sigma_{N}^{2} \sigma_{N}^{3}\right\rangle_{t} \tanh Y_{t}\right]-\frac{r}{2} \mathbf{E}\left[\left\langle\sigma_{N}^{1} \sigma_{N}^{2}\right\rangle_{t}\left(1-\tanh ^{2} Y_{t}\right)\right] .
\end{aligned}
$$

The terms $A_{3,2,1}(t)$ and $A_{3,2,2}(t)$ are respectively obtained by

$$
\begin{aligned}
A_{3,2,1}(t)= & \frac{\alpha}{2} \mathbf{E}\left[\left\langle u^{\prime}\left(S_{M, t}\right)\right\rangle_{t}\left\langle u^{\prime}\left(S_{M, 0}\right)\right\rangle_{-}\left(1-\tanh ^{2}\left(Y_{t}\right)\right)\right]-\frac{\alpha}{2} \mathbf{E}\left[\left\langle\sigma_{N}^{1} \sigma_{N}^{2} u^{\prime}\left(S_{M, t}^{1}\right)\right\rangle_{t}\left\langle u^{\prime}\left(S_{M, 0}\right)\right\rangle_{-}\left(1-\tanh ^{2}\left(Y_{t}\right)\right)\right] \\
& -\alpha \mathbf{E}\left[\left\langle\sigma_{N}\right\rangle_{t}\left\langle u^{\prime}\left(S_{M, 0}\right)\right\rangle_{-}^{2} \tanh Y_{t}\left(1-\tanh ^{2}\left(Y_{t}\right)\right)\right],
\end{aligned}
$$

and

$$
A_{3,2,2}(t)=\frac{r}{2} \mathbf{E}\left[\left(1-\tanh ^{2}\left(Y_{t}\right)\right)\right]-\frac{r}{2} \mathbf{E}\left[\left\langle\sigma_{N}^{1} \sigma_{N}^{2}\right\rangle_{t}\left(1-\tanh ^{2}\left(Y_{t}\right)\right)\right]-r \mathbf{E}\left[\left\langle\sigma_{N}\right\rangle_{t} \tanh Y_{t}\left(1-\tanh ^{2}\left(Y_{t}\right)\right)\right] .
$$

\section{Introducing a new measure}

All the preceding considerations show that, in order to prove relation (9), we should be able to expand and control any term of the form

$$
\left\langle f\left(\sigma^{1}, \ldots, \sigma^{n_{1}}\right) U_{1}\left(S_{M, t}^{1}, \ldots, S_{M, t}^{n_{1}}\right)\right\rangle_{t}\left\langle U_{2}\left(S_{M, 0}^{1}, \ldots, S_{M, 0}^{n_{2}}\right)\right\rangle_{-},
$$


for $n_{1}, n_{2} \in \mathbb{N}, U_{1}: \mathbb{R}^{n_{1}} \rightarrow \mathbb{R}, U_{2}: \mathbb{R}^{n_{2}} \rightarrow \mathbb{R}$ and $t \in(0,1]$. In this section, we will begin to develop some new tools that should allow us to handle those terms. More precisely, we will introduce some new Gibbs measures in the following way: first of all, for $t \in[0,1], \sigma \in \Sigma_{N}$, set

$$
-H_{N, M, t}^{*}(\sigma)=\sum_{k \leqslant M} u\left(S_{k, t}(\sigma)\right)+\sigma_{N} b_{t}^{*},
$$

where we recall that $Y$ is a standard Gaussian random variable independent of the remaining randomness contained in $H_{N, M, t}^{*}$, and

$$
b_{t}=(r(1-t))^{1 / 2} Y, \quad b_{t}^{*}=b_{t} \mathbf{1}_{(0,1]}(t) .
$$

Write then $G_{t}^{*}$ for the Gibbs measure related to this Hamiltonian, and notice that

$$
H_{N-1, M}(\sigma)=-\sum_{k \leqslant M} u\left(S_{k, 0}(\sigma)\right)=H_{N, M, 0}^{*}(\sigma) .
$$

For a fixed $\left(t_{1}, \ldots, t_{n}\right) \in[0,1]^{n}$, let us consider now a new Gibbs measure $G_{t_{1}, \ldots, t_{n}}^{*}$ on $\Sigma_{N}^{n}$, defined by its average on functions $f: \Sigma_{N} \rightarrow \mathbb{R}:$

$$
\langle f\rangle_{\left(t_{1}, \ldots, t_{n}\right)}:=\frac{\sum_{\sigma^{1}, \ldots, \sigma^{n}} f \exp \left(\sum_{l \leqslant n}\left(\sum_{k \leqslant M-1} u\left(S_{k, t_{l}}^{l}\right)+\sigma_{N}^{l} b_{t_{l}}^{*}\right)\right)}{\sum_{\sigma^{1}, \ldots, \sigma^{n}} \exp \left(\sum_{l \leqslant n}\left(\sum_{k \leqslant M-1} u\left(S_{k, t_{l}}^{l}\right)+\sigma_{N}^{l} b_{t_{l}}^{*}\right)\right)},
$$

where $f=f\left(\sigma^{1}, \ldots, \sigma^{n}\right)$.

Now, the terms of the form (11) can be expressed using a Gibbs measure of the form $G^{*}$ : let us consider the notation $n:=n_{1}+n_{2}$, as well as

$$
U_{1}:=U_{1}\left(S_{M, t}^{1}, \ldots, S_{M, t}^{n_{1}}\right), \quad U_{2}:=U_{2}\left(S_{M, 0}^{1}, \ldots, S_{M, 0}^{n_{2}}\right)
$$

and $f=f\left(\sigma^{1}, \ldots, \sigma^{n_{1}}\right), \widetilde{U}_{2}:=U_{2}\left(S_{M, 0}^{n_{1}+1}, \ldots, S_{M, 0}^{n}\right)$. Then, for $t \in[0,1]$,

$$
\begin{aligned}
\left\langle f U_{1}\right\rangle_{t}\left\langle U_{2}\right\rangle_{-}= & \frac{\sum_{\sigma^{1}, \ldots, \sigma^{n_{1}}} f U_{1} \exp \left(\sum_{l \leqslant n_{1}}\left[\sum_{k \leqslant M} u\left(S_{k, t}^{l}\right)+\sigma_{N}^{l}(r(1-t))^{1 / 2} Y\right]\right)}{\sum_{\sigma^{1}, \ldots, \sigma^{n_{1}}} \exp \left(\sum_{l \leqslant n_{1}}\left[\sum_{k \leqslant M} u\left(S_{k, t}^{l}\right)+\sigma_{N}^{l}(r(1-t))^{1 / 2} Y\right]\right)} \\
& \times \frac{\sum_{\sigma^{1}, \ldots, \sigma^{n_{2}}} U_{2} \exp \left(\sum_{l \leqslant n_{2}}\left[\sum_{k \leqslant M} u\left(S_{k, 0}^{l}\right)\right]\right)}{\sum_{\sigma^{1}, \ldots, \sigma^{n_{2}}} \exp \left(\sum_{l \leqslant n_{2}}\left[\sum_{k \leqslant M} u\left(S_{k, 0}^{l}\right)\right]\right)},
\end{aligned}
$$

and using the relation

$$
-H_{N, M, t}^{*}(\sigma)=-H_{N, M-1, t}^{*}\left(\sigma^{l}\right)+u\left(S_{M, t}\right),
$$

we get, for $t \in[0,1]$,

$$
\begin{aligned}
\left\langle f U_{1}\right\rangle_{t}\left\langle U_{2}\right\rangle_{-}= & \frac{\sum_{\sigma^{1}, \ldots, \sigma^{n}} f U_{1} \widetilde{U}_{2} \exp \left(-\sum_{l \leqslant n_{1}} H_{N, M, t}^{*}\left(\sigma^{l}\right)-\sum_{n_{1}<l \leqslant n} H_{N, M, 0}^{*}\left(\sigma^{l}\right)\right)}{\sum_{\sigma^{1}, \ldots, \sigma^{n}} \exp \left(-\sum_{l \leqslant n_{1}} H_{N, M, t}^{*}\left(\sigma^{l}\right)-\sum_{n_{1}<l \leqslant n} H_{N, M, 0}^{*}\left(\sigma^{l}\right)\right)} \\
= & \frac{\left\langle f U_{1} \widetilde{U}_{2} \exp \left(\sum_{l \leqslant n_{1}} u\left(S_{M, t}^{l}\right)+\sum_{n_{1}<l \leqslant n} u\left(S_{M, 0}^{l}\right)\right)\right\rangle_{(t, \ldots, t, 0, \ldots, 0)}}{\left\langle\exp \left(\sum_{l \leqslant n_{1}} u\left(S_{M, t}^{l}\right)+\sum_{n_{1}<l \leqslant n} u\left(S_{M, 0}^{l}\right)\right)\right\rangle_{(t, \ldots, t, 0, \ldots, 0)}}
\end{aligned}
$$

where in $(t, \ldots, t, 0, \ldots, 0)$ we have $n_{1}$ times $t$ and $n_{2}$ times 0 .

On the other hand, the measures $G_{t_{1}, \ldots, t_{n}}^{*}$ share some of the good properties of the original Gibbs measure, starting from the one concerning widely spread sequences of Gaussian random variables, a crucial notion that we recall briefly here (see however [4] for a detailed account on that topic).

Definition 4.1. For $n \geqslant 1$, a centered Gaussian family $\left(X_{1}, \ldots, X_{n}\right)$ is said to be widely spread if for each $l \leqslant n$ we can write

$$
X_{l}=\widehat{X}_{l}+\sum_{k \neq l} c_{l, k} X_{k}
$$


where the $c_{l, k}$ are some real coefficients, $\widehat{X}_{l}$ is independent of the random variables $X_{k}$ for $k \neq l$, and

$$
\mathbf{E}\left[\left(\widehat{X}_{l}\right)^{2}\right] \geqslant \kappa_{1},
$$

for a fixed positive constant $\kappa_{1}$.

Then, as in Lemma 3.3.3 of [4] we can prove that the measures $G_{t_{1}, \ldots, t_{n}}^{*}$ satisfy:

Lemma 4.2. Assume $u$ satisfies $(\mathrm{H} 1)$ and $(\mathrm{H} 2)$, fix $\left(t_{1}, \ldots, t_{n}\right) \in[0,1]^{n}$, and set, for any $t \in[0,1]$ and $\sigma \in \Sigma_{N}$,

$$
g_{t}(\sigma)=\frac{1}{N^{1 / 2}} \sum_{i \leqslant N-1} g_{i} \sigma_{i}+\left(\frac{t}{N}\right)^{1 / 2} g_{N} \sigma_{N},
$$

where $\left\{g_{i} ; i \leqslant N\right\}$ is a sequence of independent standard Gaussian random variables, also independent from the randomness contained in $G_{t_{1}, \ldots, t_{n}}^{*}$. Then, there exists some constants $\kappa, c_{1}>0$ such that

$$
G_{t_{1}, \ldots, t_{n}}^{*}\left(\left(\sigma^{1}, \ldots, \sigma^{n}\right) \in \Sigma_{N}^{n} ; g_{t_{1}}\left(\sigma^{1}\right), \ldots, g_{t_{n}}\left(\sigma^{n}\right) \text { is not widely spread }\right) \leqslant \kappa^{n} \exp \left(-c_{1} N\right) .
$$

Let us recall that the constant $q \in[0,1]$ has been introduced in (5) and define, for $l \leqslant n$, the quantity

$$
\theta^{l}=q^{1 / 2} W+(1-q)^{1 / 2} \xi^{l},
$$

where $W$ and $\left\{\xi^{l} ; l \geqslant 1\right\}$ are independent standard Gaussian random variables. For a fixed $t \in[0,1]$, consider also the variables

$$
g_{v, t}^{l}=v^{1 / 2} S_{M, t}^{l}+(1-v)^{1 / 2} \theta^{l},
$$

for any $v \in[0,1]$. In the sequel, we will denote by $\mathbf{E}_{\xi}$ the partial expectation with respect to the randomness contained in $\left\{\xi^{l} ; l \geqslant 1\right\}$. With Lemma 4.2 in mind, one can get the following proposition

Proposition 4.3. Assume u satisfies (H1) and (H2), and fix $\left(t_{1}, \ldots, t_{n}\right) \in[0,1]^{n}$. Then we have

1. The overlap $R_{1,2}$ self-averages into $q$ :

$$
\mathbf{E}\left[\left\langle\left(R_{1,2}-q\right)^{2}\right\rangle_{\left(t_{1}, t_{2}\right)}\right] \leqslant \frac{\kappa}{N} .
$$

2. Consider some integers $n \geqslant 1, s_{1}, \ldots, s_{n}$ and $r_{1}, \ldots, r_{n} \in\{0,1\}$ such that, setting $s=\sum_{j \leqslant n} s_{j}$ and $r=\sum_{j \leqslant n} r_{j}$, we have $s, r \leqslant 6$. Set

$$
\begin{aligned}
& V\left(x_{1}, \ldots, x_{n}\right)=\exp \left(\sum_{l \leqslant n} u\left(x_{l}\right)\right), \\
& U\left(x_{1}, \ldots, x_{n}\right)=\partial_{s_{1}, \ldots, s_{n}}^{s} V\left(x_{1}, \ldots, x_{n}\right), \\
& P\left(x_{1}, \ldots, x_{n}\right)=\prod_{j \leqslant n}\left(u^{\prime}\left(x_{l}\right)\right)^{r_{j}} .
\end{aligned}
$$

Then, for $f: \Sigma_{N}^{n} \rightarrow \mathbb{R}$ such that $|f| \leqslant 1$, we have, for any $v \in[0,1]$,

$$
\left|\frac{\left\langle f \mathbf{E}_{\xi}\left[U\left(g_{v, t_{1}}^{1}, \ldots, g_{v, t_{n}}^{n}\right)\right] P\left(S_{M-1, t_{1}}^{1}, \ldots, S_{M-1, t_{n}}^{n}\right)\right\rangle_{\left(t_{1}, \ldots, t_{n}\right)}}{\left\langle\mathbf{E}_{\xi}\left[V\left(g_{v, t_{1}}^{1}, \ldots, g_{v, t_{n}}^{n}\right)\right]\right\rangle_{\left(t_{1}, \ldots, t_{n}\right)}}\right| \leqslant \kappa\left(\mathrm{e}^{2 n D}\langle|f|\rangle+\mathrm{e}^{-c_{2} N}\right),
$$

for some positive constants $\kappa, c_{2}$.

Proof. This proof is a long and easy elaboration of the computations performed at [4, Sections 3.3 and 3.4], that we will not include here. However, notice that we have to assume here that the derivatives of $u$ are (midely) bounded up to order 12. This is due to the presence of both $U\left(g_{v, t_{1}}^{1}, \ldots, g_{v, t_{n}}^{n}\right)$ and $\widehat{P}\left(S_{M-1, t_{1}}^{1}, \ldots, S_{M-1, t_{n}}^{n}\right)$ in the left-hand side of (17). In order to treat those terms, we will separate them using Schwarz's inequality, that will bring out up to 12 derivatives of $u$. 


\section{An asymptotic expansion}

In this section, we will develop the tools that we will need in order to get an expansion of the terms of the form (11), which is a key step in the proof of Theorem 1.1. In fact, once the averages $\langle f\rangle_{\left(t_{1}, \ldots, t_{n}\right)}$ are defined, we will try, for a fixed $\left(t_{1}, \ldots, t_{n}\right)$, to find a natural path that will allow us to expand any term of the form (11), asymptotically in $N$.

Recall first that $S_{k, t}$ is defined by (3), $g_{v, t}$ by (15), $\theta^{l}$ by (14), $b_{t}$ and $b_{t}^{*}$ by (12). Observe that, using the notation introduced at Section 4, we have

$$
Y_{t}=\left(\frac{t}{N}\right)^{1 / 2} \sum_{k \leqslant M} g_{N, k} \frac{\left\langle u^{\prime}\left(S_{k, 0}\right) \exp u\left(S_{M, 0}\right)\right\rangle_{(0)}}{\left\langle\exp u\left(S_{M, 0}\right)\right\rangle_{(0)}}+b_{t} .
$$

We can turn now to the definition of the path we will use in order to get our expansion: for $v \in[0,1]$, set

$$
\bar{Y}_{v}=\left(\frac{t}{N}\right)^{1 / 2} \sum_{k \leqslant M-1} g_{N, k} \frac{\mathbf{E}_{\xi}\left\langle u^{\prime}\left(S_{k, 0}\right) \exp u\left(g_{v, 0}\right)\right\rangle_{(0)}}{\mathbf{E}_{\xi}\left\langle\exp u\left(g_{v, 0}\right)\right\rangle_{(0)}}+b_{t} .
$$

In particular it is easy to check that

$$
\begin{aligned}
& \bar{Y}_{0}=\left(\frac{t}{N}\right)^{1 / 2} \sum_{k \leqslant M-1} g_{N, k}\left\langle u^{\prime}\left(S_{k, 0}\right)\right\rangle_{(0)}+b_{t}, \\
& \bar{Y}_{1}=Y_{t}-\left(\frac{t}{N}\right)^{1 / 2} g_{N, M} \frac{\left\langle u^{\prime}\left(S_{M, 0}\right) \exp u\left(S_{M, 0}\right)\right\rangle_{(0)}}{\left\langle\exp u\left(S_{M, 0}\right)\right\rangle_{(0)}} .
\end{aligned}
$$

Using this auxiliary path, we will get the announced expansion as follows:

Proposition 5.1. Let $u$ be a function satisfying $(\mathrm{H} 1)$ and $(\mathrm{H} 2)$. Consider an integer $m$, nonnegative integers $s_{1}, \ldots, s_{m}$ with $s=\sum_{l \leqslant m} s_{l} \leqslant 2$. Set also

$$
\begin{aligned}
& V\left(x_{1}, \ldots, x_{m}\right)=\exp \sum_{l \leqslant m} u\left(x_{l}\right), \\
& U\left(x_{1}, \ldots, x_{m}\right)=\frac{\partial^{s} V}{\partial x_{1}^{s_{1}} \cdots \partial x_{m}^{s_{m}}} .
\end{aligned}
$$

Let $f$ be a function on $\Sigma_{N}^{m}$ such that $|f| \leqslant 1$ and $\Psi$ a bounded function with bounded derivatives of any order. Then, if we define, for $t_{l} \in\{0, t\}, l \leqslant m$,

$$
Q=\mathbf{E}\left[\frac{\left\langle f U\left(S_{M, t_{1}}^{1}, \ldots, S_{M, t_{m}}^{m}\right)\right\rangle_{\left(t_{1}, \ldots, t_{m}\right)}}{\left\langle V\left(S_{M, t_{1}}^{1}, \ldots, S_{M, t_{m}}^{m}\right)\right\rangle_{\left(t_{1}, \ldots, t_{m}\right)}} \Psi\left(Y_{t}\right)\right],
$$

we have

$$
Q=\mathbf{E}\left[\langle f\rangle_{\left(t_{1}, \ldots, t_{m}\right)} \Psi\left(\bar{Y}_{0}\right)\right] \mathbf{E}\left[\frac{U\left(\theta^{1}, \ldots, \theta^{m}\right)}{\mathbf{E}_{\xi} V\left(\theta^{1}, \ldots, \theta^{m}\right)}\right]+\mathcal{O}\left(N^{-1 / 2}\right)
$$

In particular, we have

$$
\mathbf{E}\left[\langle f\rangle_{\left(t_{1}, \ldots, t_{m}\right)} \Psi\left(Y_{t}\right)\right]=\mathbf{E}\left[\langle f\rangle_{\left(t_{1}, \ldots, t_{m}\right)} \Psi\left(\bar{Y}_{0}\right)\right]+\mathcal{O}\left(N^{-1 / 2}\right) .
$$

The proof of this proposition will we based on a technical lemma that we will present now. First of all, let us label some basic but useful results that we will use further on: for $l, l^{\prime} \geqslant 1, t \in[0,1]$, we have

$$
\begin{aligned}
& \mathbf{E}\left[\left(S_{M, t}^{l}\right)^{2}\right]=1+\frac{t-1}{N}, \\
& \mathbf{E}\left[S_{M, t}^{l} S_{M, t^{\prime}}^{l^{\prime}}\right]:=\Delta_{l, l^{\prime}}^{t, t^{\prime}}=R_{l, l^{\prime}}+\frac{t t^{\prime}-1}{N} \sigma_{N}^{l} \sigma_{N}^{l^{\prime}} .
\end{aligned}
$$


Lemma 5.2. Let $u$ be a function satisfying (H1) and (H2). Consider integers $n, s_{1}, \ldots, s_{n}$ with $s=\sum_{l \leqslant n} s_{l} \leqslant 6$. Consider also the functions $\widehat{U}$ and $\widehat{V}$ defined by

$$
\begin{aligned}
\widehat{U}\left(x_{1}, \ldots, x_{n}\right) & =\frac{\partial^{s} \widehat{V}}{\partial x_{1}^{s_{1}} \cdots \partial x_{n}^{s_{n}}}, \\
\widehat{V}\left(x_{1}, \ldots, x_{n}\right) & =\exp \sum_{l \leqslant n} u\left(x_{l}\right) .
\end{aligned}
$$

Let $f$ be a function on $\Sigma_{N}^{n}$ such that $|f| \leqslant 1$ and $\Phi$ a bounded function with bounded derivatives of any order. Then, for $t_{l} \in\{0, t\}, l \leqslant n, v \in[0,1]$, we have

$$
\Xi:=\mathbf{E}\left[\frac{\mathbf{E}_{\xi}\left\langle f \widehat{P} \widehat{U}\left(g_{v, t_{1}}^{1}, \ldots, g_{v, t_{n}}^{n}\right) \partial_{v} g_{v, t_{1}}^{1}\right\rangle_{\left(t_{1}, \ldots, t_{n}\right)}}{\mathbf{E}_{\xi}\left\langle\widehat{V}\left(g_{v, t_{1}}^{1}, \ldots, g_{v, t_{n}}^{n}\right)\right\rangle_{\left(t_{1}, \ldots, t_{n}\right)}} \Phi\left(\bar{Y}_{v}\right)\right]=\mathcal{O}\left(N^{-1 / 2}\right),
$$

where, $\widehat{P}=1$ or

$$
\widehat{P}=u^{\prime}\left(S_{M-1, t_{l}}^{i}\right) u^{\prime}\left(S_{M-1, t_{l^{\prime}}}^{j}\right), \quad \text { with } l, l^{\prime}, i, j \in\{1, \ldots, n\}, i \neq j .
$$

Proof of Lemma 5.2. In the sequel, we will write $\widehat{U}$ and $\widehat{V}$ instead of $\widehat{U}\left(g_{v, t_{1}}^{1}, \ldots, g_{v, t_{n}}^{n}\right)$ and $\widehat{V}\left(g_{v, t_{1}}^{1}, \ldots, g_{v, t_{n}}^{n}\right)$, respectively. We will also denote $u^{\prime}(x) \mathrm{e}^{u(x)}$ by $U_{1}(x)$. By means of (14) and (15) we can write

$$
\Xi=\Xi_{1}-\Xi_{2}-\Xi_{3}
$$

where

$$
\begin{aligned}
& \Xi_{1}=\frac{1}{2 v^{1 / 2}} \mathbf{E}\left[\frac{\mathbf{E}_{\xi}\left\langle f \widehat{P} \widehat{U} S_{M, t_{1}}^{1}\right\rangle_{\left(t_{1}, \ldots, t_{n}\right)}}{\mathbf{E}_{\xi}\langle\widehat{V}\rangle_{\left(t_{1}, \ldots, t_{n}\right)}} \Phi\left(\bar{Y}_{v}\right)\right], \\
& \Xi_{2}=\frac{1}{2(1-v)^{1 / 2}} \mathbf{E}\left[\frac{\mathbf{E}_{\xi}\left\langle f \widehat{P} \widehat{U} q^{1 / 2} W\right\rangle_{\left(t_{1}, \ldots, t_{n}\right)}}{\mathbf{E}_{\xi}\langle\widehat{V}\rangle_{\left(t_{1}, \ldots, t_{n}\right)}} \Phi\left(\bar{Y}_{v}\right)\right], \\
& \Xi_{3}=\frac{1}{2(1-v)^{1 / 2}} \mathbf{E}\left[\frac{\mathbf{E}_{\xi}\left\langle f \widehat{P} \widehat{U}(1-q)^{1 / 2} \xi^{1}\right\rangle_{\left(t_{1}, \ldots, t_{n}\right)}}{\mathbf{E}_{\xi}\langle\widehat{V}\rangle_{\left(t_{1}, \ldots, t_{n}\right)}} \Phi\left(\bar{Y}_{v}\right)\right] .
\end{aligned}
$$

We will now give a sketch of our computations: according to (20) and (21), an integration by parts argument with respect to $S_{M, t_{1}}^{1}$ or $W$ or $\xi^{1}$, and a little algebra bring out some terms of the form

$$
\widehat{\Xi}_{1} \equiv \frac{t_{1}-1}{2 N} \mathbf{E}\left[\frac{\mathbf{E}_{\xi}\left\langle f \widehat{P} \partial_{x_{1}} \widehat{U}\right\rangle_{\left(t_{1}, \ldots, t_{n}\right)}}{\mathbf{E}_{\xi}\langle\widehat{V}\rangle_{\left(t_{1}, \ldots, t_{n}\right)}} \Phi\left(\bar{Y}_{v}\right)\right],
$$

which can be handled quite easily: by Schwarz's inequality, we have

$$
\mathbf{E}\left[\frac{\mathbf{E}_{\xi}\left\langle f \widehat{P} \partial_{x_{1}} \widehat{U}\right\rangle_{\left(t_{1}, \ldots, t_{n}\right)}}{\mathbf{E}_{\xi}\langle\widehat{V}\rangle_{\left(t_{1}, \ldots, t_{n}\right)}} \Phi\left(\bar{Y}_{v}\right)\right] \leqslant\|\Phi\|_{\infty} \mathbf{E}^{1 / 2}\left[\frac{\mathbf{E}_{\xi}\left\langle f f^{*} \widehat{P} \widehat{P}^{*} \partial_{x_{1}} \widehat{U} \partial_{x_{1}} \widehat{U}^{*}\right\rangle_{\left(t_{1}, \ldots, t_{n}, t_{1}, \ldots, t_{n}\right)}}{\mathbf{E}_{\xi}\left\langle\widehat{V} \widehat{V}^{*}\right\rangle_{\left(t_{1}, \ldots, t_{n}, t_{1}, \ldots, t_{n}\right)}}\right],
$$

where

$$
f^{*}=f\left(\sigma^{n+1}, \ldots, \sigma^{n+p}\right), \quad \widehat{U}^{*}=\widehat{U}\left(g_{v, t_{1}}^{n+1}, \ldots, g_{v, t_{n}}^{2 n}\right),
$$

and $\widehat{P}^{*}, \widehat{V}^{*}$ are defined in a similar manner. Hence, using inequality (17), we get

$$
\widehat{\Xi}_{1}=\mathcal{O}\left(N^{-1}\right) \text {. }
$$

The second kind of term we have to deal with is of the type

$$
\widehat{\Xi}_{2}=\frac{1}{2} \sum_{l=2}^{n} \mathbf{E}\left[\frac{\mathbf{E}_{\xi}\left\langle f \widehat{P} \partial_{x_{l}} \widehat{U}\left(\Delta_{1, l}^{t_{1}, t_{l}}-q\right)\right\rangle_{\left(t_{1}, \ldots, t_{n}\right)}}{\mathbf{E}_{\xi}\langle\widehat{V}\rangle_{\left(t_{1}, \ldots, t_{n}\right)}} \Phi\left(\bar{Y}_{v}\right)\right],
$$

and here again, (17) and Schwarz's inequality allow us to bound $\widehat{\Xi}_{2}$ by sum of terms of the type

$$
\kappa\left(\mathbf{E}^{1 / 2}\left\langle\left|f^{2}\right|\left|\Delta_{1,2}^{t_{1}, t_{2}}-q\right|^{2}\right\rangle_{\left(t_{1}, \ldots, t_{\bar{n}}\right)}+\mathrm{e}^{-c N}\right),
$$


for some integer $\bar{n}$ and some positive constants $\kappa, c$. Then, recalling definition (21), using the fact that $|f| \leqslant 1$, and invoking inequality (16), we get $\widehat{\Xi}_{2}=\mathcal{O}\left(N^{-1 / 2}\right)$, which ends the proof.

Let us go back now to the main point of this section.

Proof of Proposition 5.1. Define the function

$$
\tau(v)=\mathbf{E}\left[\frac{\mathbf{E}_{\xi}\left\langle f U\left(g_{v, t_{1}}^{1}, \ldots, g_{v, t_{m}}^{m}\right)\right\rangle_{\left(t_{1}, \ldots, t_{m}\right)}}{\mathbf{E}_{\xi}\left\langle V\left(g_{v, t_{1}}^{1}, \ldots, g_{v, t_{m}}^{m}\right)\right\rangle_{\left(t_{1}, \ldots, t_{m}\right)}} \Psi\left(\bar{Y}_{v}\right)\right] .
$$

We have

$$
\begin{gathered}
\tau(1)=\mathbf{E}\left[\frac{\left\langle f U\left(S_{M, t_{1}}^{1}, \ldots, S_{M, t_{m}}^{m}\right)\right\rangle_{\left(t_{1}, \ldots, t_{m}\right)}}{\left\langle V\left(S_{M, t_{1}}^{1}, \ldots, S_{M, t_{m}}^{m}\right)\right\rangle_{\left(t_{1}, \ldots, t_{m}\right)}} \Psi\left(\bar{Y}_{1}\right)\right], \\
\tau(0)=\mathbf{E}\left[\langle f\rangle_{\left(t_{1}, \ldots, t_{m}\right)} \Psi\left(\bar{Y}_{0}\right)\right] \mathbf{E}\left[\frac{U\left(\theta^{1}, \ldots, \theta^{m}\right)}{\mathbf{E}_{\xi} V\left(\theta^{1}, \ldots, \theta^{m}\right)}\right] .
\end{gathered}
$$

Then, the proof of this proposition simply consists in checking that

$$
|Q-\tau(0)| \leqslant \frac{\kappa}{N^{1 / 2}} .
$$

Notice first that

$$
|Q-\tau(1)|=\left|\mathbf{E}\left[\frac{\left\langle f U\left(S_{M, t_{1}}^{1}, \ldots, S_{M, t_{m}}^{m}\right)\right\rangle_{\left(t_{1}, \ldots, t_{m}\right)}}{\left\langle V\left(S_{M, t_{1}}^{1}, \ldots, S_{M, t_{m}}^{m}\right)\right\rangle_{\left(t_{1}, \ldots, t_{m}\right)}}\left(\Psi\left(Y_{t}\right)-\Psi\left(\bar{Y}_{1}\right)\right)\right]\right| .
$$

Thus, using Schwarz's inequality, the bound (17), the fact that $\Psi$ has bounded derivatives, and the relation (19) between $\bar{Y}_{1}$ and $Y_{t}$, we get

$$
|Q-\tau(1)| \leqslant \frac{\kappa}{N^{1 / 2}}
$$

So, the proof of our proposition will easily follow from the inequality:

$$
\left|\int_{0}^{1} \tau^{\prime}(v)\right| \leqslant \frac{C}{N^{1 / 2}}
$$

that we will show now. In the sequel of the proof, we will write, for notational convenience, $U=U\left(g_{v, t_{1}}^{1}, \ldots, g_{v, t_{m}}^{m}\right)$ and $V=V\left(g_{v, t_{1}}^{1}, \ldots, g_{v, t_{m}}^{m}\right)$, and recall that we have also set $U_{1}(x)=u^{\prime}(x) \mathrm{e}^{u(x)}$. Then, we can write

$$
\tau^{\prime}(v)=\zeta_{1}(v)-\zeta_{2}(v)+\left(\frac{t}{N}\right)^{1 / 2} \sum_{k \leqslant M-1}\left(\zeta_{3}^{k}(v)-\zeta_{4}^{k}(v)\right),
$$

where

$$
\begin{aligned}
& \zeta_{1}(v)=\sum_{l \leqslant m} \mathbf{E}\left[\frac{\mathbf{E}_{\xi}\left\langle f \partial_{v} g_{v, t_{l}}^{l} \partial_{x_{l}} U\right\rangle_{\left(t_{1}, \ldots, t_{m}\right)}}{\mathbf{E}_{\xi}\langle V\rangle_{\left(t_{1}, \ldots, t_{m}\right)}} \Psi\left(\bar{Y}_{v}\right)\right], \\
& \zeta_{2}(v)=\sum_{l \leqslant m} \mathbf{E}\left[\frac{\mathbf{E}_{\xi}\left\langle f \partial_{v} g_{v, t_{l}}^{m+1} U U_{1}\left(g_{v, t_{l}}^{m+1}\right)\right\rangle_{\left(t_{1}, \ldots, t_{m}, t_{l}\right)}}{\mathbf{E}_{\xi}\left\langle V \mathrm{e}^{u\left(g_{v, t_{l}}^{m+1}\right)}\right\rangle_{\left(t_{1}, \ldots, t_{m}, t_{l}\right)}} \Psi\left(\bar{Y}_{v}\right)\right],
\end{aligned}
$$

and

$$
\begin{aligned}
& \zeta_{3}^{k}(v)=\mathbf{E}\left[g_{N, k} \frac{\mathbf{E}_{\xi}\left\langle f \partial_{v} g_{v, 0}^{m+1} u^{\prime}\left(S_{k, 0}^{m+1}\right) U U_{1}\left(g_{v, 0}^{m+1}\right)\right\rangle_{\left(t_{1}, \ldots, t_{m}, 0\right)}}{\mathbf{E}_{\xi}\left\langle V \mathrm{e}^{u\left(g_{v, 0}^{m+1}\right)}\right\rangle_{\left(t_{1}, \ldots, t_{m}, 0\right)}} \Psi^{\prime}\left(\bar{Y}_{v}\right)\right], \\
& \zeta_{4}^{k}(v)=\mathbf{E}\left[g_{N, k} \frac{\mathbf{E}_{\xi}\left\langle f \partial_{v} g_{v, 0}^{m+2} u^{\prime}\left(S_{k, 0}^{m+1}\right) \mathrm{e}^{u\left(g_{v, 0}^{m+1}\right)} U U_{1}\left(g_{v, 0}^{m+2}\right)\right\rangle_{\left(t_{1}, \ldots, t_{m}, 0,0\right)}}{\mathbf{E}_{\xi}\left\langle V \mathrm{e}^{u\left(g_{v, 0}^{m+1}\right)+u\left(g_{v, 0}^{m+2}\right)}\right\rangle_{\left(t_{1}, \ldots, t_{m}, 0,0\right)}} \Psi^{\prime}\left(\bar{Y}_{v}\right)\right] .
\end{aligned}
$$


The first two terms are of the type (22) in Lemma 5.2, and hence applying this lemma we get that

$$
\zeta_{1}(v)-\zeta_{2}(v)=\mathcal{O}\left(N^{-1 / 2}\right)
$$

To study the last two terms we have to integrate by parts again. For instance, by symmetry and integrating by parts with respect to $g_{N, M-1}$, we obtain that $\zeta_{3}^{k}(v)$ can be expressed as a function of terms of type (22). Then, applying Lemma 5.2 we can easily finish the proof of (24).

\section{Proof of Proposition 3.1}

In this section, we will see that the proof of Proposition 3.1 can easily be deduced from some nice cancellations, that we will obtain by an extensive use of Proposition 5.1.

Let us first introduce some new notation that we will use throughout this section:

$$
V_{k}\left(x_{1}, \ldots, x_{k}\right)=\exp \left(\sum_{i=1}^{k} u\left(x_{i}\right)\right), \quad U_{k}^{i, j}\left(x_{1}, \ldots, x_{k}\right)=\partial_{x_{i}, x_{j}}^{2} V_{k}\left(x_{1}, \ldots, x_{k}\right) .
$$

Recall also that definition (5) implies

$$
\mathbf{E}\left[\frac{U_{2}^{1,2}\left(\theta^{1}, \theta^{2}\right)}{\mathbf{E}_{\xi}\left(V_{2}\left(\theta^{1}, \theta^{2}\right)\right)}\right]=\mathbf{E}\left[\left|\frac{u^{\prime}\left(\theta^{1}\right) \mathrm{e}^{u\left(\theta^{1}\right)}}{\mathbf{E}_{\xi}\left(\mathrm{e}^{u\left(\theta^{1}\right)}\right)}\right|^{2}\right]=\frac{r}{\alpha} .
$$

Using the same arguments, we can also get that for $k=3,4$,

$$
\mathbf{E}\left[\frac{U_{k}^{1,2}\left(\theta^{1}, \ldots, \theta^{k}\right)}{\mathbf{E}_{\xi}\left(V_{k}\left(\theta^{1}, \ldots, \theta^{k}\right)\right)}\right]=\mathbf{E}\left[\frac{u^{\prime}\left(\theta^{1}\right) u^{\prime}\left(\theta^{2}\right) \mathrm{e}^{u\left(\theta^{1}\right)+u\left(\theta^{2}\right)}}{\mathbf{E}_{\xi}\left(\mathrm{e}^{u\left(\theta^{1}\right)+u\left(\theta^{2}\right)}\right)}\right]=\frac{r}{\alpha} .
$$

On the other hand, we will set (recall that $w=u^{\prime \prime}+\left(u^{\prime}\right)^{2}$ )

$$
\mathbf{E}\left[\frac{U_{1}^{1,1}(\theta)}{\mathbf{E}_{\xi}\left(V_{1}(\theta)\right)}\right]=\mathbf{E}\left[\frac{w\left(\theta^{1}\right) \mathrm{e}^{u\left(\theta^{1}\right)}}{\mathbf{E}_{\xi}\left(\mathrm{e}^{u\left(\theta^{1}\right)}\right)}\right]:=\delta,
$$

and observe that, for $k=2,3$,

$$
\mathbf{E}\left[\frac{U_{k}^{1,1}\left(\theta^{1}, \ldots, \theta^{k}\right)}{\mathbf{E}_{\xi}\left(V_{k}\left(\theta^{1}, \ldots, \theta^{k}\right)\right)}\right]=\mathbf{E}\left[\frac{w\left(\theta^{1}\right) \mathrm{e}^{\sum_{i=1}^{k} u\left(\theta^{i}\right)}}{\mathbf{E}_{\xi}\left(\mathrm{e}^{\sum_{i=1}^{k} u\left(\theta^{i}\right)}\right)}\right]=\delta .
$$

Using the notation introduced in Section 3 and applying Proposition 5.1, we are now able to bound all the terms appearing in the derivative of $\varphi$.

Upper bound on $\varphi_{1}^{\prime}$. We will show now how the terms appearing in Lemma 3.2 will give raise to some nice cancellations, by a direct application of (13) and Proposition 5.1. Indeed, we have for instance

$$
\begin{aligned}
A_{1,1}(t) & =\alpha \mathbf{E}\left[\frac{\left\langle\sigma_{N}^{1} \sigma_{N}^{2} U_{2}^{1,1}\left(S_{M, t}^{1}, S_{M, t}^{2}\right)\right\rangle_{(t, t)}}{\left\langle V_{2}\left(S_{M, t}^{1}, S_{M, t}^{2}\right)\right\rangle_{(t, t)}}\right]=\alpha \mathbf{E}\left[\frac{U_{2}^{1,1}\left(\theta^{1}, \theta^{2}\right)}{\mathbf{E}_{\xi}\left(V_{2}\left(\theta^{1}, \theta^{2}\right)\right)}\right] \mathbf{E}\left[\left\langle\sigma_{N}^{1} \sigma_{N}^{2}\right\rangle_{(t, t)}\right]+\mathcal{O}\left(N^{-1 / 2}\right) \\
& =\alpha \delta \mathbf{E}\left[\left\langle\sigma_{N}^{1} \sigma_{N}^{2}\right\rangle_{(t, t)}\right]+\mathcal{O}\left(N^{-1 / 2}\right) .
\end{aligned}
$$

Along the same lines, it can also be shown that $A_{1,2}(t)$ yields a contribution of

$$
-\alpha \delta \mathbf{E}\left[\left\langle\sigma_{N}^{1} \sigma_{N}^{2}\right\rangle_{(t, t)}\right]+\mathcal{O}\left(N^{-1 / 2}\right),
$$

which can be simplified with the one of $A_{1,1}(t)$. It is easily checked that the other terms defining $\varphi_{1}^{\prime}(t)$ cancel out in the same way, which gives $\varphi_{1}^{\prime}(t)=\mathcal{O}\left(N^{-1 / 2}\right)$. 
Upper bound on $\varphi_{2}^{\prime}$. Let $\Psi_{1}(y)=\left(1-\tanh ^{2}(y)\right)\left(1-3 \tanh ^{2}(y)\right)$. Applying again Proposition 5.1, we get

$$
\begin{aligned}
& A_{2,1}(t)=\alpha \mathbf{E}\left[\frac{U_{2}^{1,2}\left(\theta^{1}, \theta^{2}\right)}{\mathbf{E}_{\xi}\left(V_{2}\left(\theta^{1}, \theta^{2}\right)\right)}\right] \mathbf{E}\left[\Psi_{1}\left(\bar{Y}_{0}\right)\right]+\mathcal{O}\left(N^{-1 / 2}\right)=r \mathbf{E}\left[\Psi_{1}\left(\bar{Y}_{0}\right)\right]+\mathcal{O}\left(N^{-1 / 2}\right), \\
& A_{2,2}(t)=-r \mathbf{E}\left[\Psi_{1}\left(\bar{Y}_{0}\right)\right]+\mathcal{O}\left(N^{-1 / 2}\right) .
\end{aligned}
$$

Hence, $\varphi_{2}^{\prime}(t)=\mathcal{O}\left(N^{-1 / 2}\right)$.

Upper bound on $\varphi_{3}^{\prime}$. Set $\Psi_{2}(y)=\tanh ^{2}(y)$ Here again,we will expand the terms appearing in Lemma 3.4, thanks to Proposition 5.1, and look for some cancellations. An example of computation is

$$
\begin{aligned}
A_{3,1,1,1}(t) & =\frac{\alpha}{2} \mathbf{E}\left[\frac{\left\langle\sigma_{N} U_{1}^{1,1}\left(S_{M, t}\right)\right\rangle_{(t)}}{\left\langle V_{1}\left(S_{M, t}\right)\right\rangle_{(t)}} \Psi_{2}\left(Y_{t}\right)\right]=\frac{\alpha}{2} \mathbf{E}\left[\frac{U_{1}^{1,1}(\theta)}{\mathbf{E}_{\xi}\left(V_{1}(\theta)\right)}\right] \mathbf{E}\left[\left\langle\sigma_{N}\right\rangle_{(t)} \Psi_{2}\left(\bar{Y}_{0}\right)\right]+\mathcal{O}\left(N^{-1 / 2}\right) \\
& =\frac{\alpha}{2} \delta \mathbf{E}\left[\left\langle\sigma_{N}\right\rangle_{(t)} \Psi_{2}\left(\bar{Y}_{0}\right)\right]+\mathcal{O}\left(N^{-1 / 2}\right),
\end{aligned}
$$

and following these steps, we obtain

$$
\sum_{i=1}^{4} A_{3,1,1, i}(t)=\frac{\alpha}{2} \delta \mathbf{E}\left[\left\langle\sigma_{N}\right\rangle_{(t)} \Psi_{2}\left(\bar{Y}_{0}\right)\right]-\frac{r}{2} \mathbf{E}\left[\left\langle\sigma_{N}\right\rangle_{(t)} \Psi_{2}\left(\bar{Y}_{0}\right)\right]+\mathcal{O}\left(N^{-1 / 2}\right)
$$

The same kind of expansions also yield

$$
\sum_{i=1}^{5} A_{3,1,2, i}(t)=\frac{\alpha}{2} \delta \mathbf{E}\left[\left\langle\sigma_{N}\right\rangle_{(t)} \Psi_{2}\left(Y_{0}\right)\right]-\frac{r}{2} \mathbf{E}\left[\left\langle\sigma_{N}\right\rangle_{(t)} \Psi_{2}\left(Y_{0}\right)\right]+\mathcal{O}\left(N^{-1 / 2}\right),
$$

which cancels out with the contribution of $\sum_{i=1}^{4} A_{3,1,1, i}(t)$. Finally, a similar kind of argument shows that

$$
A_{3,2,1}(t)-A_{3,2,2}(t)=\mathcal{O}\left(N^{-1 / 2}\right) .
$$

Then, from (26), (27) and (28) we get that $\varphi_{3}^{\prime}(t)=\mathcal{O}\left(N^{-1 / 2}\right)$, which ends the proof of Proposition 3.1.

\section{Proof of Theorem 1.1}

Once Proposition 3.1 is proved, our main theorem will be shown along some more or less classical lines. However, we still have to prove here a technical lemma:

Lemma 7.1. Let $\langle\cdot\rangle_{-}$be the Gibbs average introduced at Proposition 3.1. Then, there exists a constant $\kappa>0$ such that

$$
\mathbf{E}\left[\left(\left\langle\sigma_{1}\right\rangle-\left\langle\sigma_{1}\right\rangle_{-}\right)^{2}\right] \leqslant \frac{\kappa}{N^{1 / 2}} .
$$

Proof. For the proof of this lemma, we will need to consider again a slight variation of the Gibbs measures defined up to now: recall that $b_{t}$ has been introduced by Eq. (12). Then, for $n \geqslant 1$, a test function $f: \Sigma_{N}^{n} \rightarrow \mathbb{R}$ and $\left(t_{1}, \ldots, t_{n}\right) \in$ $[0,1]^{n}$, set

$$
\langle f\rangle_{\left(t_{1}, \ldots, t_{n}\right)}:=\frac{\sum_{\sigma^{1}, \ldots, \sigma^{n}} f \exp \left(\sum_{l \leqslant n} H_{N, M, t_{l}}\left(\sigma^{l}\right)\right)}{\sum_{\sigma^{1}, \ldots, \sigma^{n}} \exp \left(\sum_{l \leqslant n} H_{N, M, t_{l}}\left(\sigma^{l}\right)\right)}=\frac{\sum_{\sigma^{1}, \ldots, \sigma^{n}} f \exp \left(\sum_{l \leqslant n}\left(\sum_{k \leqslant M} u\left(S_{k, l^{l}}^{l}\right)+\sigma_{N}^{l} b_{t_{l}}\right)\right)}{\sum_{\sigma^{1}, \ldots, \sigma^{n}} \exp \left[\sum_{l \leqslant n}\left(\sum_{k \leqslant M} u\left(S_{k, l^{l}}^{l}\right)+\sigma_{N}^{l} b_{t_{l}}\right)\right]} .
$$

Notice that $\langle f\rangle_{(t, \ldots, t)}^{\circ}=\langle f\rangle_{t}$, but in general, $\langle f\rangle_{(0)}^{\circ}$ and $\langle f\rangle_{-}$do not coincide, because of the presence of the term $b_{t}$ in the first one. However, observe also that if $f^{-}: \Sigma_{N}^{n} \rightarrow \mathbb{R}$ does not depend on the last coordinates $\left\{\sigma_{N}^{l} ; l \leqslant n\right\}$, then

$$
\left\langle f^{-}\right\rangle_{(0, \ldots, 0)}^{\circ}=\left\langle f^{-}\right\rangle_{-} .
$$

In particular,

$$
\mathbf{E}\left[\left(\left\langle\sigma_{1}\right\rangle-\left\langle\sigma_{1}\right\rangle_{-}\right)^{2}\right]=\mathbf{E}\left[\left(\left\langle\sigma_{1}\right\rangle_{(1)}^{\circ}-\left\langle\sigma_{1}\right\rangle_{(0)}^{\circ}\right)^{2}\right] .
$$


For $t \in[0,1]$, set now

$$
\psi(t)=\mathbf{E}\left[\left(\left\langle\sigma_{1}\right\rangle_{(t)}^{\circ}-\left\langle\sigma_{1}\right\rangle_{(0)}^{\circ}\right)^{2}\right]=\psi_{1}(t)-2 \psi_{2}(t)+\psi_{3},
$$

with

$$
\psi_{1}(t)=\mathbf{E}\left[\left\langle\sigma_{1}^{1} \sigma_{1}^{2}\right\rangle_{(t, t)}^{\circ}\right], \quad \psi_{2}(t)=\mathbf{E}\left[\left\langle\sigma_{1}^{1} \sigma_{1}^{2}\right\rangle_{(t, 0)}^{\circ}\right], \quad \psi_{3}=\mathbf{E}\left[\left\langle\sigma_{1}^{1} \sigma_{1}^{2}\right\rangle_{(0,0)}^{\circ}\right]
$$

Obviously, $\psi(0)=0$. Then in order to finish our proof it suffices to show that $\psi_{1}^{\prime}(t)$ and $\psi_{2}^{\prime}(t)$ are of order $N^{-1 / 2}$. Indeed, $\psi_{1}^{\prime}(t)$ can be computed through Proposition 2.2. Then, along the same lines as in [4, Chapter 3], one can show that $\psi_{1}^{\prime}(t)$ is of order $N^{-1 / 2}$ for any $t \in[0,1]$. The term $\psi_{2}^{\prime}(t)$ can be treated by the same kind of argument: in fact, it can be computed like in Proposition 2.2, except that we will differentiate only with respect to the first replica. We obtain

$$
\psi_{2}^{\prime}(t)=L_{1}(t)+L_{2}(t)+L_{3}(t)
$$

with

$$
L_{1}(t)=\frac{\alpha}{2} \mathbf{E}\left[\left\langle\left.\sigma_{1}^{1} \sigma_{1}^{2} w\left(S_{M, t}^{1}\right)\right|_{(t, 0)} ^{\circ}\right]-\frac{\alpha}{2} \mathbf{E}\left[\left\langle\left.\sigma_{1}^{1} \sigma_{1}^{2} w\left(S_{M, t}^{3}\right)\right|_{(t, 0, t)} ^{\circ}\right],\right.\right.
$$

where $w(x)=u^{(2)}(x)+\left(u^{\prime}(x)\right)^{2}$,

$$
L_{2}(t)=\alpha \mathbf{E}\left[\left\langle\left.\sigma_{1}^{1} \sigma_{1}^{2} \sigma_{N}^{3} \sigma_{N}^{4} u^{\prime}\left(S_{M, t}^{3}\right) u^{\prime}\left(S_{M, t}^{4}\right)\right|_{(t, 0, t, t)} ^{\circ}\right]-\alpha \mathbf{E}\left[\left\langle\left.\sigma_{1}^{1} \sigma_{1}^{2} \sigma_{N}^{1} \sigma_{N}^{3} u^{\prime}\left(S_{M, t}^{1}\right) u^{\prime}\left(S_{M, t}^{3}\right)\right|_{(t, 0, t)} ^{\circ}\right],\right.\right.
$$

and

$$
L_{3}(t)=-r\left(\mathbf{E}\left[\left\langle\sigma_{1}^{1} \sigma_{1}^{2} \sigma_{N}^{3} \sigma_{N}^{4}\right\rangle_{(t, 0, t, t)}^{\circ}\right]-\mathbf{E}\left[\left\langle\sigma_{1}^{1} \sigma_{1}^{2} \sigma_{N}^{1} \sigma_{N}^{3}\right\rangle_{(t, 0, t)}^{\circ}\right]\right) .
$$

Then, $\psi_{2}^{\prime}(t)$ can be bounded by $\kappa N^{-1 / 2}$ using again the same kind of arguments as in [4, Chapter 3].

Let us turn now to the main aim of this section.

Proof of Theorem 1.1. This proof is now a slight variation of [4, Section 2.4], and we sketch it here for sake of completeness.

Step 1 . Since $\alpha>0, r$ is bounded from below by a constant $r_{0}>0$ (see relation (5)). Set

$$
z=\frac{\sum_{k \leqslant M} g_{N, k}\left\langle u^{\prime}\left(S_{k, 0}\right)\right\rangle_{-}}{s},
$$

with

$$
s^{2}=\sum_{k \leqslant M}\left\langle u^{\prime}\left(S_{k, 0}\right)\right\rangle_{-}^{2} .
$$

Then it can be shown, as in [4, Section 2.4], that $z$ is a standard Gaussian random variable independent of the random variables $\left\{g_{i, k} ; i \leqslant N-1, k \leqslant M\right\}$. Furthermore, following again the steps of [4, Lemma 2.4.14], it can be shown that

$$
\mathbf{E}\left[\left(\left\langle\sigma_{N}\right\rangle-\tanh \left(r^{1 / 2} z\right)\right)^{2}\right] \leqslant \frac{\kappa}{N^{1 / 2}} .
$$

Indeed, using Proposition 3.1, the Lipschitz property of the function $x \mapsto \tanh (x)$ and some easy algebraic manipulations, we obtain for some positive constant $\kappa$,

$$
\begin{aligned}
\mathbf{E}\left[\left(\left\langle\sigma_{N}\right\rangle-\tanh \left(r^{1 / 2} z\right)\right)^{2}\right] & \leqslant \kappa \mathbf{E}\left[\left(\sum_{k \leqslant M}\left(\frac{1}{N^{1 / 2}}-\frac{r^{1 / 2}}{s}\right) g_{N, k}\left\langle u^{\prime}\left(S_{k, 0}\right)\right\rangle_{-}\right)^{2}\right]+\mathcal{O}\left(N^{-1 / 2}\right) \\
& =\kappa \mathbf{E}\left[\left(\frac{1}{N^{1 / 2}}-\frac{r^{1 / 2}}{s}\right)^{2} \sum_{k \leqslant M}\left\langle u^{\prime}\left(S_{k, 0}\right)\right\rangle_{-}^{2}\right]+\mathcal{O}\left(N^{-1 / 2}\right) \\
& \leqslant \frac{\kappa}{r} \mathbf{E}\left[\left(\frac{s^{2}}{N}-r\right)^{2}\right]+\mathcal{O}\left(N^{-1 / 2}\right) .
\end{aligned}
$$


Then notice that, using again the symmetry among the $S_{k, t}$, we can write

$$
\mathbf{E}\left[\left(\frac{s^{2}}{N}-r\right)^{2}\right]=\mathbf{E}\left[\left(\frac{s^{2}}{N}-r\right)\left(\alpha\left\langle u^{\prime}\left(S_{M, 0}^{1}\right) u^{\prime}\left(S_{M, 0}^{2}\right)\right\rangle_{-}-r\right)\right]=T_{1}+T_{2}+T_{3}+r^{2}
$$

where

$$
\begin{aligned}
T_{1} & :=\alpha \frac{M-1}{N} \mathbf{E}\left[\left\langle u^{\prime}\left(S_{M, 0}^{1}\right) u^{\prime}\left(S_{M, 0}^{2}\right)\right\rangle_{-}\left\langle u^{\prime}\left(S_{M-1,0}^{1}\right) u^{\prime}\left(S_{M-1,0}^{2}\right)\right\rangle_{-}\right], \\
T_{2} & :=\frac{\alpha}{N} \mathbf{E}\left[\left\langle u^{\prime}\left(S_{M, 0}^{1}\right) u^{\prime}\left(S_{M, 0}^{2}\right)\right\rangle_{-}\left\langle u^{\prime}\left(S_{M, 0}^{1}\right) u^{\prime}\left(S_{M, 0}^{2}\right)\right\rangle_{-}\right], \\
T_{3} & :=-2 \alpha r \mathbf{E}\left[\left\langle u^{\prime}\left(S_{M, 0}^{1}\right) u^{\prime}\left(S_{M, 0}^{2}\right)\right\rangle_{-}\right] .
\end{aligned}
$$

Clearly, $T_{2}=\mathcal{O}\left(N^{-1 / 2}\right)$. On the other hand, using Proposition 5.1 we have

$$
T_{3}=-2 \alpha r \mathbf{E}\left[\frac{U_{2}^{1,2}\left(\theta^{1}, \theta^{2}\right)}{\mathbf{E}_{\xi}\left(V_{2}\left(\theta^{1}, \theta^{2}\right)\right)}\right]=-2 r^{2} .
$$

So, in order to finish the proof of (29) it is enough to check that $T_{1}=r^{2}+\mathcal{O}\left(N^{-1 / 2}\right)$. Although this result cannot be obtained as a direct consequence of our Proposition 5.1, it can be proved following exactly the same ideas and steps as in the proofs of this proposition and Lemma 5.2. For the sake of simplicity we will not repeat here such proof.

Step 2. Along the same lines as in [4, Theorem 2.4.12], the proof can be done by induction over $m$ on the system of $N-1$ spins. Our hypothesis of induction yields that

$$
\sum_{i \leqslant m} \mathbf{E}\left[\left(\left\langle\sigma_{i}\right\rangle_{-}-\tanh \left(r_{-}^{1 / 2} z_{i}\right)\right)^{2}\right] \leqslant \frac{\kappa}{N^{1 / 2}},
$$

where $\left\{z_{i} ; i \leqslant m\right\}$ is a collection of independent standard Gaussian random variables depending only on the variables $\left\{g_{i, j}\right\}_{i \leqslant N-1}$, and $r_{-}$is the solution to (5) when $\alpha$ is replaced by $\alpha_{-}=M /(N-1)$. Notice that it is easily seen that

$$
\left|r-r_{-}\right| \leqslant \frac{\kappa}{N^{1 / 2}} \text {. }
$$

Then the random variable $z_{m+1}=z$ defined in the first step of the proof, is independent of the random variables $\left\{z_{i}, i \leqslant m\right\}$. The desired conclusion is then obtained by (30), (31) and Lemma 7.1.

\section{References}

[1] X. Bardina, D. Márquez-Carreras, C. Rovira, S. Tindel, The p-spins interaction model with external field, Potential Anal. 21 (4) (2004) $311-362$.

[2] J. Hertz, A. Krogh, R. Palmer, Introduction to the Theory of Neural Computation, Addison-Wesley Publishing Company, 1991.

[3] M. Shcherbina, B. Tirozzi, Rigorous solution of the Gardner problem, Comm. Math. Phys. 234 (3) (2003) 383-422.

[4] M. Talagrand, Spin Glasses: A Challenge for Mathematicians, Springer, Berlin, 2003. 